\title{
CORRESPONDENCE
}

\section{Does PDE4 inhibition improve alveolarisation in hyperoxia-exposed immature rodents?}

\section{To the Editors:}

A recent European Respiratory Journal article by WoYDA et al. [1] discussed the effect of a phosphodiesterase (PDE) 4 inhibitor, cilomilast, on alveolarisation in mouse pups exposed to hyperoxia. WoYDA et al. [1] claimed in the title and concluded in the text that this treatment had the beneficial effect of preventing hyperoxia-induced impairment of alveolarisation, and proposed that PDE4 inhibition could represent a potential new therapy for bronchopulmonary dysplasia. We think that this conclusion overstated the study design and the obtained results. Although we used a different inhibitor (rolipram), our recently published study indicated that PDE4 inhibition did not enhance alveolarisation in rat pups exposed to hyperoxia, and may even directly affect alveolarisation in rat pups exposed to room air [2].

The latter finding leads to the first and major criticism that can be aimed at the article of WOYDA et al. [1], namely the lack of an appropriate control group to study the effect of the PDE4 inhibitor on normal alveolarisation as evaluated by administering it to air-exposed pups. As a consequence of the lack of this control group, two of the main effects of PDE4 inhibition in rodent pups that we reported (impairment of weight gain and disturbed alveolarisation) were not evaluated by WOYDA et al. [1]. The effect on weight gain may have been less than that observed by us [2] and others [3], as WoYDA et al. [1] studied mice instead of rats and administered the PDE4 inhibitor later in life. But studying the effects of PDE4 inhibition on normal alveolarisation may have revealed crucial changes and is clearly missing.

A second major concern about the study of WoYDA et al. [1] is an overstatement of the reported data. Figure 5 of their paper shows an obvious rarefaction of alveoli under hyperoxia, but the figure does not show any improvement of alveolarisation after PDE4 inhibition. The morphometric determinations used are very simplistic and rough compared with data that can be obtained with these methods and usually published in the field [4]. Moreover, WOYDA et al. [1] did not demonstrate any biologically significant beneficial effect of PDE4 inhibition on hyperoxiainduced impairment of alveolarisation; although the mean linear intercept was statistically different between hyperoxia and hyperoxia+cilomilast groups, the difference was extremely weak (a $4 \%$ reduction only!) and the mean linear intercept remained considerably increased as compared to that in animals maintained under normoxia (plus 78\% and plus 70\% in hyperoxia and hyperoxia+cilomilast groups, respectively). This minute variation, even if statistically significant, cannot be considered biologically relevant and does not allow the authors to claim a beneficial effect of cilomilast. The total airspace changes were in the same range of very weak variations (roughly $118 \%$ of the baseline value under hyperoxia and $114 \%$ with cilomilast). Consistent with this very weak, if actual, effect on alveolarisation, there was no effect on dynamic compliance. Performing an in-depth analysis of alveolarisation in rat pups in our previous study according to the criteria established by WEIBEL and CRUZORVIVE [4], we found no beneficial effect of PDE4 inhibition on alveolarisation. Furthermore, PDE4 inhibition under air induced a decrease in alveolar surface area and radial alveolar count [2], the interpretation of which was rendered difficult by the impairment of weight gain of pups treated with PDE4 inhibitors, because undernutrition and impaired weight gain can modify lung morphometric parameters.

Considering these remarks and the present state of development of phosphodiesterase 4 inhibitors, to claim for enhanced alveolarisation by phosphodiesterase 4 inhibition in a bronchopulmonary dysplasia model appears to be an overstatement. It is at least premature, if not erroneous and dangerous, to propose phosphodiesterase 4 inhibition as a new treatment of bronchopulmonary dysplasia on the basis of data reported in the paper by WoYDA et al. [1].

\section{Méhats*,\#, ${ }^{*}$, J. Bourbon ${ }^{\uparrow,+}$ and P-H. Jarreau ${ }^{*, \#, \uparrow, \S}$}

*Université Paris Descartes, Faculté de Médecine, "Institut National de la Santé et de la Recherche Médicale (INSERM) U767, "PremUP, ${ }^{\S}$ Assistance Publique-Hôpitaux de Paris, Service de Médecine Néonatale de Port-Royal, Paris, and 'INSERM U955, équipe 13, Créteil, France.

\section{STATEMENT OF INTEREST}

None declared.

\section{REFERENCES}

1 Woyda K, Koebrich S, Reiss I, et al. Inhibition of phosphodiesterase 4 enhances lung alveolarisation in neonatal mice exposed to hyperoxia. Eur Respir J 2009; 33: 861-870.

2 Mehats C, Franco-Montoya ML, Boucherat O, et al. Effects of phosphodiesterase 4 inhibition on alveolarization and hyperoxia toxicity in newborn rats. PLOS ONE 2008; 3: e3445.

3 de Visser YP, Walther FJ, Laghmani EH, van Wijngaarden S, Nieuwland K, Wagenaar GT. Phosphodiesterase 4 inhibition attenuates pulmonary inflammation in neonatal lung injury. Eur Respir J 2007; 31: 633-644.

4 Weibel ER, Cruz-Orive LM. Morphometric methods. In: Crystal RG, West JB, Weibel ER, Barnes PJ, eds. The Lung: Scientific Foundations. Philadelphia, Lippincott-Raven, 1997; pp. 333-344. 